\title{
COMMUTATIVITY AND DECOMPOSITION FOR NEAR RINGS
}

\author{
HAMZA A. S. ABUJABAL
}

\begin{abstract}
Let $R$ be a distributively generated (d.g) near ring satisfy one of the following
\end{abstract} conditions.

(*) For each $x, y$ in $R$, there exists a positive integer $n=n(x, y)$ such that $x y=(y x)^{n}$.

(**) For each $x, y$ in $R$, there exist positive integers $m=m(x, y)$ and $n=n(x, y)$ for which $x y=y^{m} x^{n}$.

In [2], Bell proved the commutativity of $R$ satisfying (*) or (**) under appropriate additional hypothesis. In this paper, we generalize the above properties for wider class of near rings known as D-near rings. Also we provide an example for justification of our results. Furthermore, we give a decomposition Theorem for near rings satisfying $(* *)$.

\section{Some Commutativity Theorems for Near Rings}

In [6], Ligh and Luh introduced the notion of D-ring as follows:

Definition 1. A near ring $R$ is called a D-near ring if every non-zero homomorphic image $S$ of $R$ satisfies the following conditions:

$\left(C_{1}\right) S$ has a non-zero right distributive element.

$\left(C_{2}\right)(S,+)$ is abelian implies that $(S,+,$.$) is a ring.$

All rings and distributively generated $(d . g)$ near rings are examples of D-near rings.

The following example shows that $\mathrm{D}$-near rings are generalizations of $d . g$ near rings.

Example 1 . Let $R=\{0, x, y, z, u, v\}$ with addition and multiplication tables, defined as follows:

Received June 18, 1996.

1991 Mathematics Subject Classification. 16U80.

Key words and phrases. Near rings, D-near rings, distributively generated near rings, zero symmetric, zero commutative, commutativity, annihilators, orthogonal sum and decomposition of near rings. 


\begin{tabular}{c|cccccc}
+ & 0 & $x$ & $y$ & $z$ & $u$ & $v$ \\
\hline 0 & 0 & $x$ & $y$ & $z$ & $u$ & $v$ \\
$x$ & $\mathrm{x}$ & 0 & $v$ & $u$ & $z$ & $y$ \\
$y$ & $\mathrm{y}$ & $u$ & 0 & $v$ & $x$ & $z$ \\
$z$ & $z$ & $v$ & $u$ & $o$ & $y$ & $x$ \\
$u$ & $\mathrm{u}$ & $y$ & $z$ & $x$ & $v$ & 0 \\
$v$ & $\mathrm{v}$ & $z$ & $x$ & $y$ & 0 & $u$
\end{tabular}

\begin{tabular}{c|cccccc}
$\cdot$ & 0 & $x$ & $y$ & $z$ & $u$ & $v$ \\
\hline 0 & 0 & 0 & 0 & 0 & 0 & 0 \\
$x$ & 0 & $x$ & $x$ & $x$ & 0 & 0 \\
$y$ & 0 & $x$ & $z$ & $y$ & $v$ & $u$ \\
$t$ & 0 & $x$ & $y$ & $z$ & $u$ & $v$ \\
$u$ & 0 & 0 & 0 & 0 & 0 & 0 \\
$v$ & 0 & 0 & 0 & 0 & 0 & 0
\end{tabular}

Then $R$ is a $D$-near ring which has a unique left identity $z$ with $u z=0=v z$. This indicates that $z$ is not a right identity. By virtue of [7, Theorem 3.2], if a d.g near ring has a unique left identity, then it is also a right identity. Thus $R$ is not a $d . g$ near ring. This shows that the class of D-near rings is larger than the class of $d . g$ near rings.

Let $R$ be a $D$-near ring. In this section, we study the commutativity of $R$ satisfying one of the following conditions:

(*) For each $x, y$ in $R$, there exists a positive integer $n=n(x, y)$ such that $x y=(y x)^{n}$.

(**) For each $x, y$ in $R$, there exist positive integers $m=m(x, y)$ and $n=n(x, y)$ for which $x y=y^{m} x^{n}$.

Definition 2. A near ring $R$ is called zero-symmetric if $0 x=0$ for all $x \in R$, that is left distributive gives $x 0=0$.

Definition 3. A near ring $R$ is called zero-commutative if $x y=0$ implies that $y x=0$ for $x, y \in R$.

First, we recall [1, Lemma 1], [1, Lemma 2] and [3, Lemma 3].

Lemma 1 . Let $R$ be a zero-symmetric near ring satisfying the following conditions:

(a) For each $x$ in $R$, there exists an integer $n=n(x)>1$ such that $x^{n}=x$.

(b) Every non-trivial homomorphic image of $R$ contains a non-zero central idempotent. Then $(R,+)$ is abelian.

Lemma 2. Let $R$ be a zero-symmetric near ring with no non-zero nilpotent elements. Then the following conditions hold.

(i) Every distributive idempotent is central.

(ii) For every idempotent $e$ and every element $y$ in $R, e y^{2}=(e y)^{2}$.

(iii) If $R$ has a multiplicative identity element, then all idempotent elements are central.

Lemma 3. Let $R$ be a near ring which is zero commutative. Then

(i) If $a, b \in R$ such that $a b=0$, then arb $=0$ for all $r \in R$.

(ii) The annihilator of any non-empty subset of $R$ is an ideal. 
(iii) The set of all nilpotent elements is an ideal if it is a subgroup of the additive group $R^{+}$of $R$.

First, we prove the following lemma.

Lemma 4. Let $R$ be a near ring satisfying (*) or (**). Then the idempotent elements of $R$ are central.

Proof. Let $R$ satisfies (*), and let $e$ be an idempotent element of $R$. If $x \in R$, then there exist integers $p=p(e, x) \geq 1$ and $n=n(x, e) \geq 1$ such that $x e=(e x)^{n}$ and $e x=(x e)^{p}$. Multiplying by $e$ on the left of the first and right of the second, we get exe $=e(e x)^{n}=(e x)^{n}=x e$ and $e x e=(x e)^{p} e=(x e)^{p}=e x$. Thus, $e x=x e$. Therefore, the idempotent elements of $R$ are central.

Now, let $R$ satisfies $(* *)$ and $e$ be an idempotent element of $R$. Then there exist integers $r=r(x, e) \geq 1$ and $s=s(x, e) \geq 1$ such that $x e=e^{r} x^{s}=e x^{s}$. Thus exe $=e x^{s}=$ $x e$. Also, for some $m=m(e, x) \geq 1$ and $n=n(e, x) \geq 1$, we have $e x=x^{m} e^{n}=x^{m} e$. Thus exe $=x^{m} e=e x$. So exe $=e x$. Hence $e x=x e$ for all $x$ in $R$. Therefore, the idempotent elements of $R$ are central.

Lemma 5. Let $R$ be a zero-symmetric D-near ring. If for each $x$ in $R$, there exists a positive integer $m=m(x)>1$ such that $x^{m}=x$, then $R$ is a commutative ring.

Proof. By the definition of D-near ring, every non-zero homomorphic image of $R$ contains a non-zero distributive element. If $x$ is a non-zero distributive element with $m=m(x)>1$ such that $x^{m}=x$, then $x^{m-1}=e$, that is $x^{m-1}$ is a distributive idempotent. In view of Lemma $2(\mathrm{i}), x^{m-1}$ is a distributive central idempotent, because $R$ has no non-zero nilpotent elements. By Lemma $1,(R,+)$ is abelian. But $R$ is a D-near ring. Hence $R$ is a ring. By a well-known result of Jacobson [5], $R$ is a commutative ring.

Now, we are in a position to prove our main results.

Theorem 1. Let $R$ be a D-near ring satisfying (*). Thus $R$ is commutative.

Proof. If $n=n(x, y)=1$, then $x y=y x$. Thus $R$ is commutative. Assume that $n=n(x, y)>1$ and $R$ satisfies the property $(*)$. First, we show that $x y=0$. By $(*)$, we have $y x=(x y)^{n}=0$. Therefore, $R$ is zero-commutative. Thus the left and the right annihilator of $R$ coincide. Let $A$ be an an annihilator of $R$. By Lemma 3(ii), $A$ becomes an ideal. Let $a$ in $R$ with $a^{2}=0$. Given any $x \in R$ we have $a x a=0$ because $a(a x)=0$ and $R$ is zero-commutative. By assumption $a x=(x a)^{n}$ for some $n=n(a, x)>1$. Therefore $a x=x(a x a)(x a)^{n-2}=0$ and so $a \in A$. Since the homomorphic image $R / A$ of $R$ is a D-near ring, take positive integers $p$ and $q$ such that $x^{2}=x^{p+q}$. Thus $x\left(x^{p+q-1}-x\right)=0$. But $R$ is zero-commutative. So $\left(x^{p+q-1}-x\right) x=0$. Hence $x\left(x^{p+q-1}-x\right) x^{p+q-1}=0$. Therefore, $\left(x^{p+q-1}-x\right)^{2}=0$. This shows that $x^{p+q-1}-x \in A$. By Lemma $5, R / A$ is a commutative ring. Hence $x(x y-y x)=0$, and so $x^{2} y=x y x$ for all $x \in R$. But 
$x^{2}=x^{p+q}$. Thus $x^{p+q-2}=e$ is idempotent and so is central by Lemma 4 . Hence

$$
\begin{aligned}
y x^{2} & =x^{p+q-2} y x^{2} \\
& =x^{p+q-3} x y x^{2} \\
& =x^{p+q-3}(x y x) x \\
& =x^{p+q-3} x^{2} y x \\
& =x^{p+q-2} x y x \\
& =x^{p+q-2} x^{2} y \\
& =x^{2} y .
\end{aligned}
$$

So

$$
y x^{2}=x^{2} y=x y x \text { for all } x, y \in R .
$$

By our hypothesis $(*)$, we can write

$$
x y=(y x)^{n} \text { where } n=n(x, y)>1 .
$$

Similarly, for each pair of elements $y, x$ in $R$, there exists an integer $r=r(y, x)>1$ such that $y x=(x y)^{r}$. This implies that $(x y)^{r n}=x y$. Now, we have

$$
\begin{aligned}
x y & =(x y)^{r n} \\
& =\left((x y)^{r}\right)^{n} \\
& =(\underbrace{x y \cdot x y \cdot x y \cdots x y}_{r-\text { times }})^{n} \\
& =((x y x) \underbrace{y x \cdot y x \cdot y x \cdots y x}_{(r-2)-\text { times }} y)^{n}
\end{aligned}
$$

Repeated (1) continuously, we get

$$
\begin{aligned}
x y & =(y x^{2} \underbrace{y x y x \cdots y x}_{(r-2)-\text { times }} y)^{n} \\
& =((y x)^{2} \underbrace{x y x y \cdots x y}_{(r-2)-\text { times }})^{n} \\
& =\left((y x)^{r-2} y x^{2} y\right)^{n} \\
& =\left((y x)^{r-2} y x y x\right)^{n} \\
& =\left((y x)^{r}\right)^{n} \\
& =(x y)^{n} \\
& =y x .
\end{aligned}
$$

Therefore, $R$ is commutative. 
Theorem 2. Let $R$ be a D-near ring satisfying (**). Then $R$ is commutative.

Proof. Let $R$ satisfies $(* *)$ and let $n=n(x, y) \geq 1$ and $m=m(x, y) \geq 1$. Then it is easy to check that $R$ is zero-commutative. Then by $(* *)$, we get

$$
y x=x^{n} y^{m}=\underbrace{x x \cdots(x}_{n \text {-times }} \underbrace{y) y \cdots y}_{m-\text { times }}=0 .
$$

Let $x, y \in R$ with $x y=0$. Then $R$ is zero commutative.

Using the same argument as in the proof of Theorem 1, we get

$$
x^{2} y=x y x=y x^{2} \text { for all } x, y \in R .
$$

This implies that for any $s \geq 2$, we can write

$$
x^{s} y=y x^{s} \text { for all } x, y \in R .
$$

Now by (**), we have $x y=y^{m} x^{n}$. Let $y=x^{p} y^{q}$ for some positive integers $p, q, m$ and $n$. Using (2), we obtain

$$
\begin{aligned}
x y & =y^{m-1} y x x^{n-1} \\
& =y^{m-1} x^{p} y^{q} x^{n-1} \\
& =y^{m+q-1} x^{n+p-1}
\end{aligned}
$$

Thus

$$
x y=y^{m+q-1} x^{n+p-1} .
$$

Furthermore, by using (2) and (3), we get

$$
\begin{aligned}
x y & =x^{n+p-1} y^{m+q-1} \\
& =x^{p-1} x^{n} y^{m} y^{q-1} \\
& =x^{p-1} y^{m} x^{n} y^{q-1} \\
& =x^{p} y^{q} \quad=y x .
\end{aligned}
$$

Hence $x y=y x$. Therefore, $R$ is commutative.

\section{A Decomposition Theorem for Near Rings}

In [4], Bell and Ligh established the direct sum decomposition for rings satisfying the properties $x y=(x y)^{2} f(x y)$ and $x y=(y x)^{2} f(y x)$, where $f(X) \in \mathbb{Z}[X]$, the polynomial ring over $\mathbb{Z}$. Furtheremore, in [4], they remarked that in case of near rings the analogous results do not give direct sum decomposition. The authors of [4], defined a weaker condition of orthogonal sum as follows.

Definition 4. A near ring $R$ is an orthogonal sum of subnear rings $M$ and $N$ denoted by $R=M \oplus N$, if $M N=N M=(0)$ and each element of $R$ has a unique representation in the form $m+n$ such that $m \in M$ and $n \in N$.

In this paper, we consider the near ring property: 
$(* * *)$ For each $x, y$ in a near ring $R$, there exist positive integers $m=m(x, y) \geq 1$ and $n=n(x, y)>1$ for which $x y=y^{m} x^{n}$. Indeed, we prove the following result.

Theorem 3. Let $R$ be a near ring satisfying $(* * *)$. Then the set $N$ of all nilpotent elements of $R$ is a subnear ring with trivial multiplication. Indeed $N$ is an ideal in $R$. If $M=\left\{x \in R \mid x^{n}=x\right.$, for a positive integer $\left.n(x)>1\right\}$, then $M$ is a subnear ring of $R$ with $(M,+)$ is abelian. Furthermore, $R=M \oplus N$.

Before proving our decomposition result for near rings, we state the following lemma.

Lemma 6 [4]. Let $R$ be a near ring with idempotent elements are multiplicative central, and let $e$ and $f$ be any idempotent element of $R$. Then there exists an idempotent element $g$ such that $g e=e$ and $g f=f$.

Proof of Theorem 3. Let $R$ satisfies $(* * *)$. Then it is easy to check that $R$ is necessarily zero-symmetric as well as zero-commutative. Suppose that $a \in N$ and $x \in R$. Then there exist integers $m_{1}=m_{1}(a, x) \geq 1$, and $n_{1}=n_{1}(a, x)>1$ such that

$$
a x=x^{m_{1}} a^{n_{1}} .
$$

Now, select $m_{2}=m_{2}\left(x^{m_{1}}, a^{n_{1}}\right) \geq 1$ and $n_{2}=n_{2}\left(x^{m_{1}}, a^{n_{1}}\right)>1$ such that

$$
x^{m_{1}} a^{n_{1}}=a^{n_{1} n_{2}} x^{m_{1} m_{2}} .
$$

Combining (4) and (5), we obtain

$$
a x=a^{n_{1} n_{2}} x^{m_{1} m_{2}}
$$

Using the same argument as above for arbitrary $q$, such that the integers $m_{1} \geq 1, m_{2} \geq 1$, $\ldots, m_{q} \geq 1$ and $n_{1}>1, n_{2}>1, \ldots, n_{q}>1$. But $a \in N$. So $a^{n_{1} n_{2} \ldots n_{q}}=0$ for sufficiently large $q$. Thus $a x=0$. But $R$ is zero-commutative. Then the nilpotent elements of $R$ annihilate $R$ on both sides, so $N R=R N=(0)$. This implies that $N^{2}=(0)$ and $N \subseteq Z(R)$, the center of $R$. Further, let $a, b \in N$ such that $a^{s^{\prime}}=0$ and $b^{t^{\prime}}=0$ for all $s^{\prime} \geq 1$ and $t^{\prime}>1$. Then $(a-b)^{s^{\prime}+t^{\prime}}=0$, that is $a-b \in N$. By Lemma 3 (iii), we get $N$ is an ideal of $R$.

Let $r \in R$ and let $s>1, t>1$ be integers such that $r^{s+t}=r^{2}$. So we have $r=r-r^{s+t-1}-r^{s+t-1}$. Because $r\left(r-r^{s+t-1}\right)=0$ and $R$ is zero-commutative. So we get $\left(r-r^{s+t-1}\right) r=0$ and $\left(r-r^{s+t-1}\right) r^{s+t-1}=0$. Hence $\left(r-r^{s+t-1}\right)^{2}=0$ and $r-r^{s+t-1} \in N$. Also, we have

$$
\begin{aligned}
\left(r^{s+t-1}\right)^{s+t-1} & =r^{(s+t-1)(s+t-1)} \\
& =r^{(s+t-2)(s+t)} \cdot r \\
& =\left(r^{s+t}\right)^{s+t-2} \cdot r \\
& =\left(r^{2}\right)^{s+t-2} \cdot r \\
& =\left(r^{s+t-2}\right)^{2} \cdot r .
\end{aligned}
$$


Since $r^{s+t-2}$ is idempotent, $\left(r^{s+t-1}\right)^{s+t-1}=r^{s+t-1}$ for $s+t-1>1$ and $r^{s+t-1} \in M$.

Next, we show that $M$ is a subnear ring of $R$. Let $u, v \in M$ and let $l=l(u) \geq 1$, $k=k(v)>1$ be integers such that $u^{l}=u$ and $v^{k}=v$. Then $e=u^{l-1}$ and $f=v^{k-1}$ are idemoptent elements such that $e u=u$ and $f v=v$. Thus

$$
u v=e u f v=e f u v=u v e f=(e f)^{m}(u v)^{n}
$$

for some integers $m=m(u v, e f) \geq 1$ and $n=n(u v, e f)>1$. Hence, we can write

$$
u v=e f(u v)^{n}=(u v)^{n} .
$$

This implies that $u v \in M$. Since $R / N$ has the property $x^{j(x)}=x$ for an integer $j(x)>1$. So we have an integer $i>1$ such that

$$
(u-v)^{i}=(u-v+a) \text { for } a \in N .
$$

Using Lemma 6, we choose an idempotent $h$ for which $h e=e$ and $h f=f$ such that $h u=u$, and $h v=v$. Multiplying (6) by $h$, we get $(u-v)^{i}=u-v \in M$. This implies that $M$ is a subnear ring.

By Lemma 1, $(M,+)$ is abelian. It is obvious to see that $M \cap N=(0)$. Now, let $x_{1}+y_{1}=x_{2}+y_{2}$ for $x_{1}, x_{2} \in M$ and $y_{1}, y_{2} \in N$. Then $x_{1}-x_{2}=y_{2}-y_{1}$. Since $x_{1}-x_{2} \in M$ and $y_{2}-y_{1} \in N$. This implies that $x_{1}-x_{2}=y_{2}-y_{1} \in M \cap N=(0)$. Thus $x_{1}=x_{2}$ and $y_{1}=y_{2}$. Therefore, $R=M \oplus N$.

\section{References}

[1] H. E. Bell, "Near rings in which each element is a power of itself," Bull. Austral. Math. Soc., 2 (1970), 363-368.

[2] H. E. Bell, "Certain near rings are rings II," Internat. J. Math and Math. Sci., 9 (1986), 267-272.

[3] H. E. Bell, "Certain near rings are rings," J. London Math. Soc., 4 (1971), 267-270.

[4] H. E. Bell, "Some decomposition theorems for periodic rings and near rings," Math. J. Okayama Univ., 31 (1989), 93-99.

[5] N. Jacobson, "Structure of rings," Amer. Math. Soc. Colloq. Pub., 37 Providence, (1956).

[6] S. Ligh and J. Luh, "Some commutativity theorems for rings and near rings," Acta Math. Acad. Sci. Hungar., 28 (1976), 19-23.

[7] S. Ligh, "On distributively generated near rings," Proc. Edinb. Math. Soc., 16 (1969), 239-243.

[8] M. A. Quadri, M. Ashraf and A. Ali, "Certain conditions under which near rings are rings," Bull. Austral. Math. Soc., 42 (1990), 91-94.

Department of Mathematics, Faculty of Science, King Abdulaziz University, P. O. Box 31464, Jeddah 21497, Saudi Arabia. 